\title{
Asymmetric Synthesis of 1,2,3-Trisubstituted \\ Cyclopentanes and Cyclohexanes as Key \\ Components of Substance P Antagonists
}

Jeffrey T. Kuethe, * Audrey Wong, Jimmy Wu, Ian W. Davies, Peter G. Dormer,

Christopher J. Welch, Michael C. Hillier, David L. Hughes, and Paul J. Reider

Department of Process Research, Merck \& Co., Inc., P.O. Box 2000, Rahway, NJ 07065,

USA

Jeffrey_Kuethe@merck.com

General Procedure for Suzuki-Cross Coupling of 2-Bromo-3-alkoxycyclopenten-1-ones and 2-Bomo-3-alkoxy-cyclohexen-1-ones with Arylboronic Acids. To a solution of the appropriate bromide (10 mmol), $\mathrm{K}_{2} \mathrm{CO}_{3}(20 \mathrm{mmol})$, and the appropriate arylboronic acid $(13 \mathrm{mmol})$ was added $\mathrm{Pd}_{2}(\mathrm{dba})_{3}(0.10 \mathrm{mmol})$ and $\mathrm{PPh}_{3}(0.20$ mmol). The resulting mixture was heated at reflux for $12 \mathrm{~h}$, cooled to $20{ }^{\circ} \mathrm{C}$, and then washed with $25 \mathrm{~mL}$ of $0.33 \mathrm{M} \mathrm{K}_{3} \mathrm{PO}_{4}$. The resulting toluene layer was concentrated under reduced pressure and the residue was purified by flash silica gel chromatography.

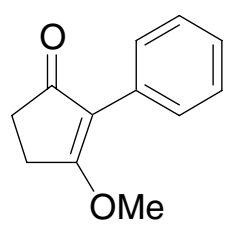


3-Methoxy-2-phenyl-2-cyclopenten-1-one. Colorless solid (82\%): $\mathrm{mp}$ 83-83 • C (MTBE/hexane); ${ }^{1} \mathrm{H}$ NMR $\left(\mathrm{CDCl}_{3}, 400 \mathrm{MHz}\right) \delta 2.59$ (m, 2H), 2.80 (m, 2H), $4.00(\mathrm{~s}, 3 \mathrm{H})$, $7.27(\mathrm{~m}, 1 \mathrm{H}), 7.37(\mathrm{~m}, 2 \mathrm{H}), 7.72(\mathrm{~m}, 2 \mathrm{H}) ;{ }^{13} \mathrm{C} \mathrm{NMR}\left(\mathrm{CDCl}_{3}, 100 \mathrm{MHz}\right) \delta 24.7,33.8$, 57.0, 118.8, 127.1, 128.0, 128.5, 132.1, 184.7, 203.0; Anal. Calcd. For $\mathrm{C}_{12} \mathrm{H}_{12} \mathrm{O}_{2}$ : C, 76.57; H, 6.43. Found: C, 76.19; H, 6.37.

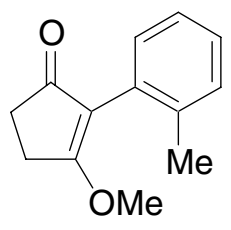

3-Methoxy-2-(2-tolyl)-2-cyclopenten-1-one. Colorless solid (99\%): mp 87-88 •C (MTBE/hexane); ${ }^{1} \mathrm{H}$ NMR $\left(\mathrm{CDCl}_{3}, 400 \mathrm{MHz}\right) \delta 2.19(\mathrm{~s}, 3 \mathrm{H}), 2.63(\mathrm{~m}, 2 \mathrm{H}), 2.81$ (m, 2H), $3.82(\mathrm{~s}, 3 \mathrm{H}), 7.21(\mathrm{~m}, 1 \mathrm{H}), 7.27(\mathrm{~m}, 3 \mathrm{H}) ;{ }^{13} \mathrm{C} \mathrm{NMR}\left(\mathrm{CDCl}_{3}, 100 \mathrm{MHz}\right) \delta$ 20.2, 26.0, 33.9, 57.8, 120.7, 125.6, 128.1, 130.2, 130.7, 132.2, 137.4, 184.4, 203.5; Anal. Calcd. For $\mathrm{C}_{13} \mathrm{H}_{14} \mathrm{O}_{2}$ : C, 77.20; H, 6.98. Found: C, 77.01; H, 6.93.

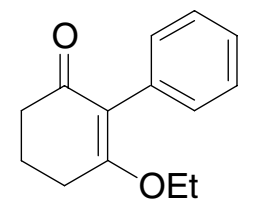

3-Ethoxy-2-phenyl-2-cyclohexen-1-one. Colorless solid (65\%): mp 83-84 • C (MTBE/hexane); ${ }^{1} \mathrm{H} \mathrm{NMR}\left(\mathrm{CDCl}_{3}, 400 \mathrm{MHz}\right) \delta 1.21(\mathrm{t}, 3 \mathrm{H}, J=7.0 \mathrm{~Hz}), 2.10(\mathrm{~m}, 2 \mathrm{H})$, $2.50(\mathrm{~m}, 2 \mathrm{H}), 2.70(\mathrm{t}, 2 \mathrm{H}, J=6.3 \mathrm{~Hz}), 3.96(\mathrm{q}, 2 \mathrm{H}, J=7.0 \mathrm{~Hz}), 7.19(\mathrm{~m}, 2 \mathrm{H}), 7.26(\mathrm{~m}$, 1H), $7.35(\mathrm{~m}, 2 \mathrm{H}) ;{ }^{13} \mathrm{C} \mathrm{NMR}\left(\mathrm{CDCl}_{3}, 100 \mathrm{MHz}\right) \delta 15.0,20.6,26.3,36.8,64.4,120.5$, 126.5, 127.6, 130.7, 133.5, 172.5, 197.5; Anal. Calcd. For $\mathrm{C}_{14} \mathrm{H}_{16} \mathrm{O}_{2}$ : C, 77.75; H, 7.46. Found: C, 77.41; H, 7.42. 


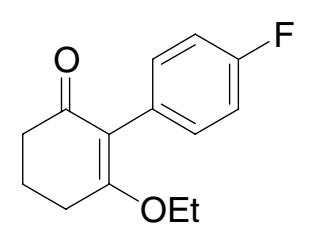

3-Ethoxy-2-(4-fluorophenyl)-cyclohexen-1-one. Colorless solid (76\%): mp 87$88 \bullet \mathrm{C}(\mathrm{MTBE} / \mathrm{h} e x a n e) ;{ }^{1} \mathrm{H} \mathrm{NMR}\left(\mathrm{CDCl}_{3}, 400 \mathrm{MHz}\right) \delta 1.22(\mathrm{t}, 3 \mathrm{H}, J=7.0 \mathrm{~Hz}), 2.09(\mathrm{~m}$, 2H), $2.48(\mathrm{t}, 2 \mathrm{H}, H=6.7 \mathrm{~Hz}), 2.70(\mathrm{t}, 2 \mathrm{H}, J=6.2 \mathrm{~Hz}), 4.00(\mathrm{q}, 2 \mathrm{H}, J=7.0 \mathrm{~Hz}), 7.01(\mathrm{~m}$, 2H), $7.16(\mathrm{~m}, 2 \mathrm{H}) ;{ }^{13} \mathrm{C} \mathrm{NMR}\left(\mathrm{CDCl}_{3}, 100 \mathrm{MHz}\right) \delta 15.2,20.8,26.4,37.0,64.5,114.5(\mathrm{~d}$, $J=20 \mathrm{~Hz}), 119.8,129.3,132.5,161.6(\mathrm{~d}, J=240 \mathrm{~Hz}), 172.4,197.3$; Anal. Calcd. For $\mathrm{C}_{14} \mathrm{H}_{15} \mathrm{FO}_{2}$ : C, 71.78; H, 6.45. Found: C, 71.48; H, 6.40.

General Procedure for Coupling of 1,3-Cyclopentanediones and 1,3Cyclohexanediones with Aryl Chlorides. Method A in 1,4-dioxane. To a $100 \mathrm{~mL}$ round bottom flask was sequentially added $\mathrm{Pd}(\mathrm{OAc})_{2} \quad(0.25 \mathrm{mmol})$, 2-(di-tbutylphosphino)biphenyl $(0.55 \mathrm{mmol})$, the appropriate 1,3 -dione $(25.24 \mathrm{mmol})$, and powdered $\mathrm{K}_{3} \mathrm{PO}_{4}(50.5 \mathrm{mmol})$. The resulting mixture was degassed $(3 \mathrm{X})$ by vacuum $/ \mathrm{N}_{2}$ back fills. The vessel was then charged with $26 \mathrm{~mL}$ of 1,4-dioxane and the appropriate aryl chloride $(32.78 \mathrm{mmol})$. The vessel was degassed $(3 \mathrm{X})$ with vacuum/ $\mathrm{N}_{2}$ back fills. The resulting slurry was heated to reflux for $12 \mathrm{~h}$, cooled to $\mathrm{rt}$, and water $(75 \mathrm{~mL})$ was added. To the homogeneous solution was added $6 \mathrm{~N} \mathrm{HCl}$ to adjust the $\mathrm{pH}$ to 1 and the slurry aged for $2.5 \mathrm{~h}$. The slurry was then filtered and the cake washed with 1 bed volume of water and 1 bed volume of toluene. The solid was then dried under vacuum at $60 \cdot \mathrm{C}$ for $48 \mathrm{~h}$. An analytical sample was obtained by recrystallization from acetone/hexane. 


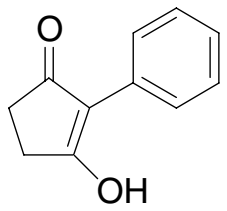

2-Phenyl-1,3-cyclopentanedione. White solid (96\%); mp: 233 •C (decomp, acetone/hexane); ${ }^{1} \mathrm{H}$ NMR (d - py, $\left.400 \mathrm{MHz}\right) \delta 2.54(\mathrm{~s}, 4 \mathrm{H}), 7.31(\mathrm{~m}, 1 \mathrm{H}), 7.51(\mathrm{~m}, 2 \mathrm{H})$, $8.54(\mathrm{~m}, 2 \mathrm{H}), 12.5$ (br s, $1 \mathrm{H}) ;{ }^{13} \mathrm{C} \mathrm{NMR}\left(\mathrm{d}_{5}-\mathrm{py}, 100 \mathrm{MHz}\right) \delta 32.2,116.0,127.3,129.1$, 129.3, 134.5, 195.5; Anal. Calcd. For $\mathrm{C}_{11} \mathrm{H}_{10} \mathrm{O}_{2}$ : C, 75.84; H, 5.79. Found: C, 75.49; H, 5.86.

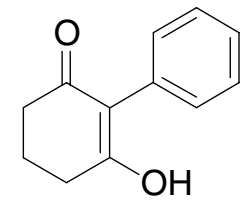

2-Phenyl-1,3-cyclohexanedione. White solid (65\%); $\mathrm{mp}: 147-148 \quad \mathrm{C}$ (acetone/hexane); ${ }^{1} \mathrm{H}$ NMR (d - py, $\left.400 \mathrm{MHz}\right) \delta 1.93(\mathrm{~m}, 2 \mathrm{H}), 2.64(\mathrm{t}, 4 \mathrm{H}, J=6.3 \mathrm{~Hz})$, $7.26(\mathrm{~m}, 1 \mathrm{H}), 7.43(\mathrm{~m}, 2 \mathrm{H}), 7.71(\mathrm{~m}, 2 \mathrm{H}), 11.90$ (br s, $1 \mathrm{H}) ;{ }^{13} \mathrm{C}$ NMR $\left(\mathrm{d}_{5}-\mathrm{py}, 100 \mathrm{MHz}\right)$ $\delta$ 21.6, 34.6, 118.5, 127.0, 128.0, 131.0, 185.1; Anal. Calcd. For $\mathrm{C}_{12} \mathrm{H}_{12} \mathrm{O}_{2}$ : C, 76.57; H, 6.43. Found: C, 76.24; H, 6.42.

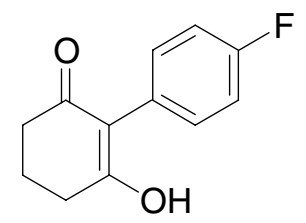

2-(4-fluorophenyl)-1,3-cyclohexanedione. White Solid (75\%); mp: 203-205 •C (acetone/hexane); ${ }^{1} \mathrm{H}$ NMR (d 5 -py, $\left.400 \mathrm{MHz}\right) \delta 1.93(\mathrm{~m}, 2 \mathrm{H}), 2.64(\mathrm{~m}, 4 \mathrm{H}), 7.20(\mathrm{~m}$, 2H), $7.65(\mathrm{~m}, 2 \mathrm{H}), 12.1$ (br s, 1H); ${ }^{13} \mathrm{C} \mathrm{NMR}\left(\mathrm{d}_{5}-\mathrm{py}, 100 \mathrm{MHz}\right) \delta 22.0,34.9,115.5(\mathrm{~d}, J=$ 
$20 \mathrm{~Hz}), 117.7,132.3,134.6,162.7(\mathrm{~d}, J=240 \mathrm{~Hz}), 185.7$; Anal. Calcd. For $\mathrm{C}_{12} \mathrm{H}_{11} \mathrm{FO}_{2}$ : C, 69.89; H, 5.38. Found: C, 69.81; H, 5.15.

\section{General Procedure for Bromination of 3-Alkoxy-2-substituted-cyclopenten-} 1-ones and 3-Alkoxy-2-substituted-cyclohexen-1-ones. To a solution of the appropriate 3-alkoxy-2-substituted-cyclopenten-1-one or cyclohexen-1-one (10 mmol) in $25 \mathrm{~mL}$ of 1,2-dichloroethane was added $\mathrm{PBr}_{3}(15 \mathrm{mmol})$. The resulting mixture was heated to reflux for $1 \mathrm{~h}$, cooled to $20 \bullet \mathrm{C}$, and poured over cracked ice. The organic layer was separated, washed with sat. aqueous $\mathrm{NaHCO}_{3}$, and dried over $\mathrm{MgSO}_{4}$. The solvent was removed under reduced pressure and the residue purified over a plug of silica gel.

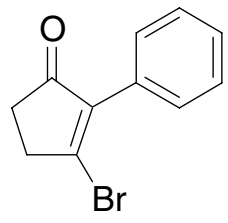

3-Bromo-2-phenyl-2-cyclopenten-1-one. White solid (65\%): mp 39-40 • C (MTBE/hexane); ${ }^{1} \mathrm{H} \mathrm{NMR}\left(\mathrm{CDCl}_{3}, 400 \mathrm{MHz}\right) \delta 2.73(\mathrm{~m}, 2 \mathrm{H}), 3.11(\mathrm{~m}, 2 \mathrm{H}), 7.46(\mathrm{~m}$, $5 \mathrm{H}) ;{ }^{13} \mathrm{C} \mathrm{NMR}\left(\mathrm{CDCl}_{3}, 100 \mathrm{MHz}\right) \delta 35.8,36.4,128.3,128.8,129.0,130.1,143.2,156.0$, 202.3; Anal. Calcd. For $\mathrm{C}_{11} \mathrm{H}_{9}$ BrO: C, 55.72; H, 3.83. Found: C, 55.63; H, 3.71 .

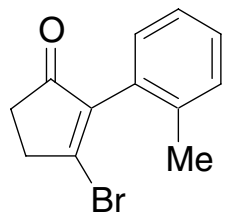

3-Bromo-2-(2-tolyl)-2-cyclopenten-1-one. White solid (62\%); mp 59-60 •C (MTBE/hexane); ${ }^{1} \mathrm{H}$ NMR $\left(\mathrm{CDCl}_{3}, 400 \mathrm{MHz}\right) \delta 2.18$ (s, 3H), 2.75 (m, 2H), 3.14 (m, 2H), $7.05(\mathrm{~d}, 1 \mathrm{H}, J=7.5 \mathrm{~Hz}), 7.27(\mathrm{~m}, 3 \mathrm{H}) ;{ }^{13} \mathrm{C} \mathrm{NMR}\left(\mathrm{CDCl}_{3}, 100 \mathrm{MHz}\right) \delta 20.0,35.9,36.6$, 
125.9, 129.1, 129.5, 130.5, 130.7, 136.8, 158.0, 202.4; Anal. Calcd. For $\mathrm{C}_{12} \mathrm{H}_{11} \mathrm{BrO}$ : C, 57.39; H, 4.42. Found: C, 57.08, H, 4.37.

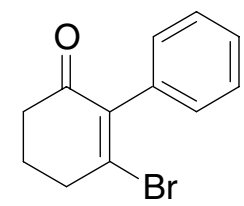

3-Bromo-2-phenyl-2-cyclohexen-1-one. Colorless solid (64\%); mp 77-78 • C (MTBE/hexane); ${ }^{1} \mathrm{H} \mathrm{NMR}\left(\mathrm{CDCl}_{3}, 400 \mathrm{MHz}\right) \delta 2.15$ (m, 2H), 2.61 (t, 2H, $J=6.8 \mathrm{~Hz}$ ), $3.07(\mathrm{t}, 2 \mathrm{H}, J=6.2 \mathrm{~Hz}), 7.20(\mathrm{~d}, 2 \mathrm{H}, J=6.7 \mathrm{~Hz}), 7.40(\mathrm{~m}, 3 \mathrm{H}) ;{ }^{13} \mathrm{C} \mathrm{NMR}\left(\mathrm{CDCl}_{3}, 100\right.$ MHz) $\delta 22.9,37.7,38.0,127.9,128.0,129.5,136.1,142.0,148.2$, 194.6; Anal. Calcd. For $\mathrm{C}_{12} \mathrm{H}_{11} \mathrm{BrO}$ : C, 57.40; H, 4.42. Found: $\mathrm{C}, 57.09 ; \mathrm{H}, 4.27$.

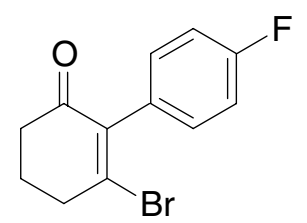

3-Bromo-2-(4-fluorophenyl)-2-cyclohexen-1-one. Colorless solid (66\%); mp 76-77 • C (MTBE/hexane); ${ }^{1} \mathrm{H}$ NMR $\left(\mathrm{CDCl}_{3}, 400 \mathrm{MHz}\right) \delta 2.17(\mathrm{~m}, 2 \mathrm{H}), 2.62(\mathrm{t}, 2 \mathrm{H}, J=$ $\left.6.8 \mathrm{~Hz}), 3.07(\mathrm{t}, 2 \mathrm{H}, J=6.1 \mathrm{~Hz}), 7.10(\mathrm{~m}, 4 \mathrm{H}) ;{ }^{13} \mathrm{C} \mathrm{NMR}\left(\mathrm{CDCl}_{3}, 100 \mathrm{MHz}\right)\right) \delta 23.0$, 37.8, 38.2, $115.2(\mathrm{~d}, J=20 \mathrm{~Hz}), 131.6,132.0,162.6(\mathrm{~d}, J=250 \mathrm{~Hz}), 194.7$; Anal. Calcd. For $\mathrm{C}_{12} \mathrm{H}_{10} \mathrm{BrFO}$ : C, 53.56; $\mathrm{H}, 3.75$. Found: $\mathrm{C}, 53.58 ; \mathrm{H}, 3.43$. 

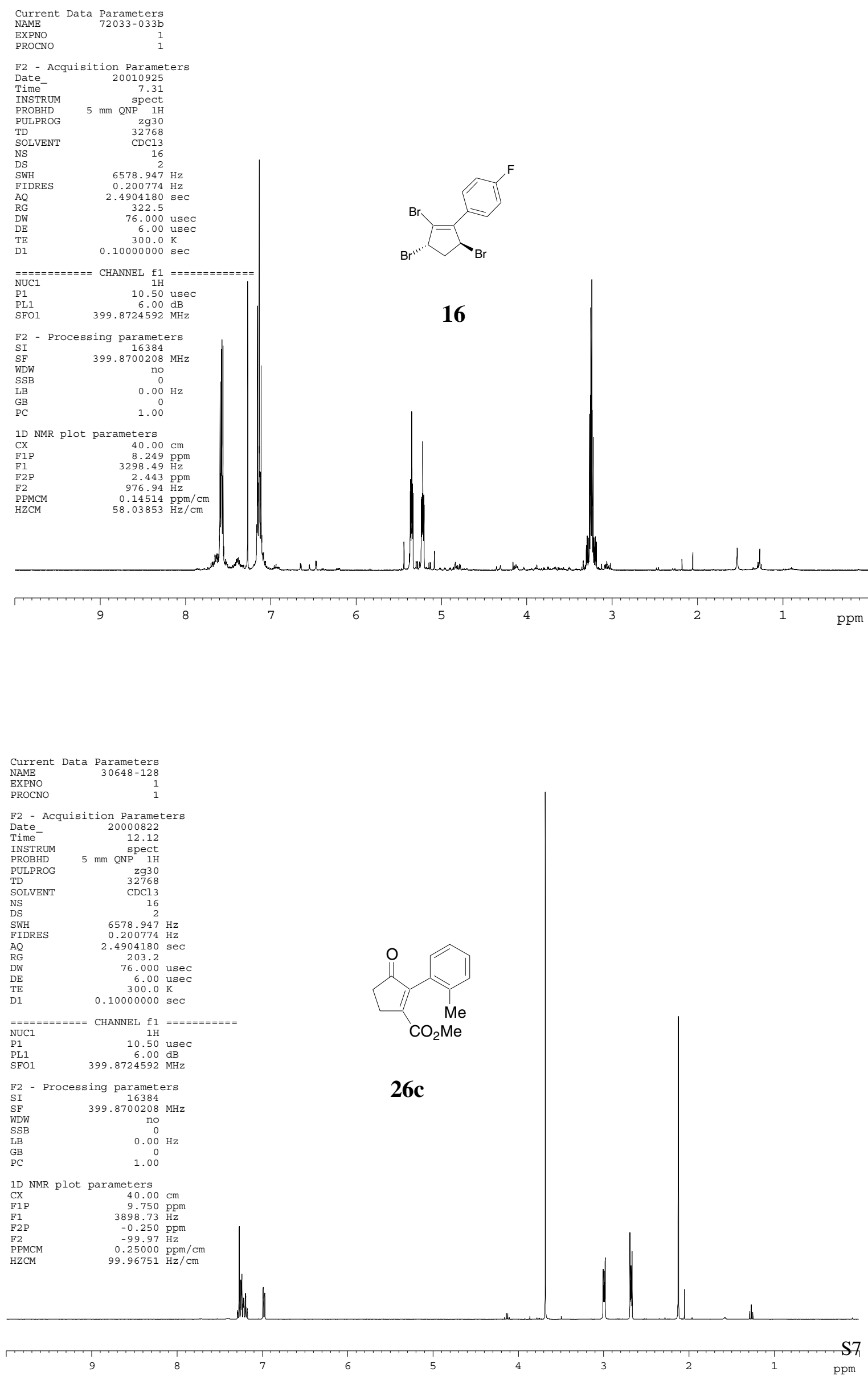

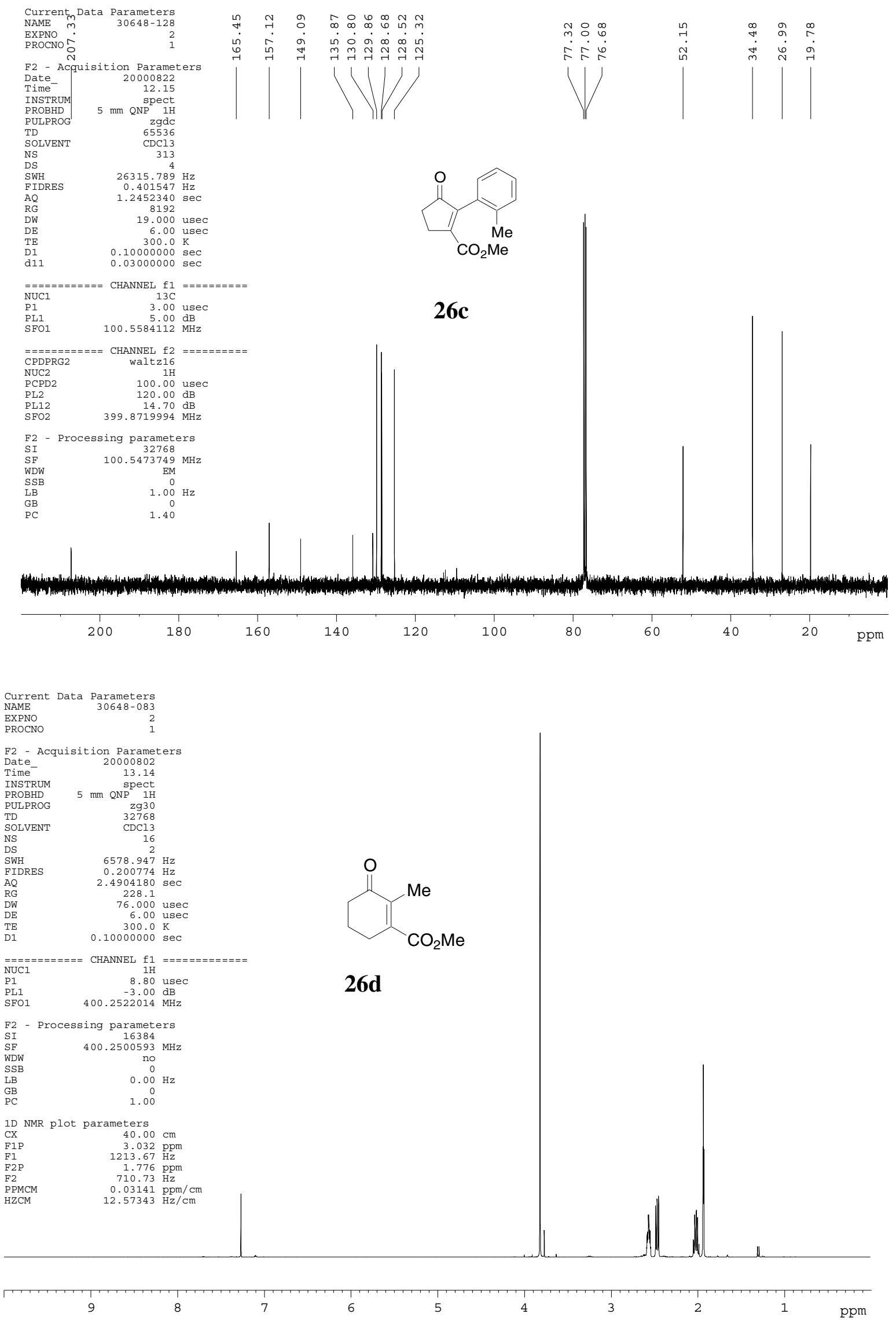


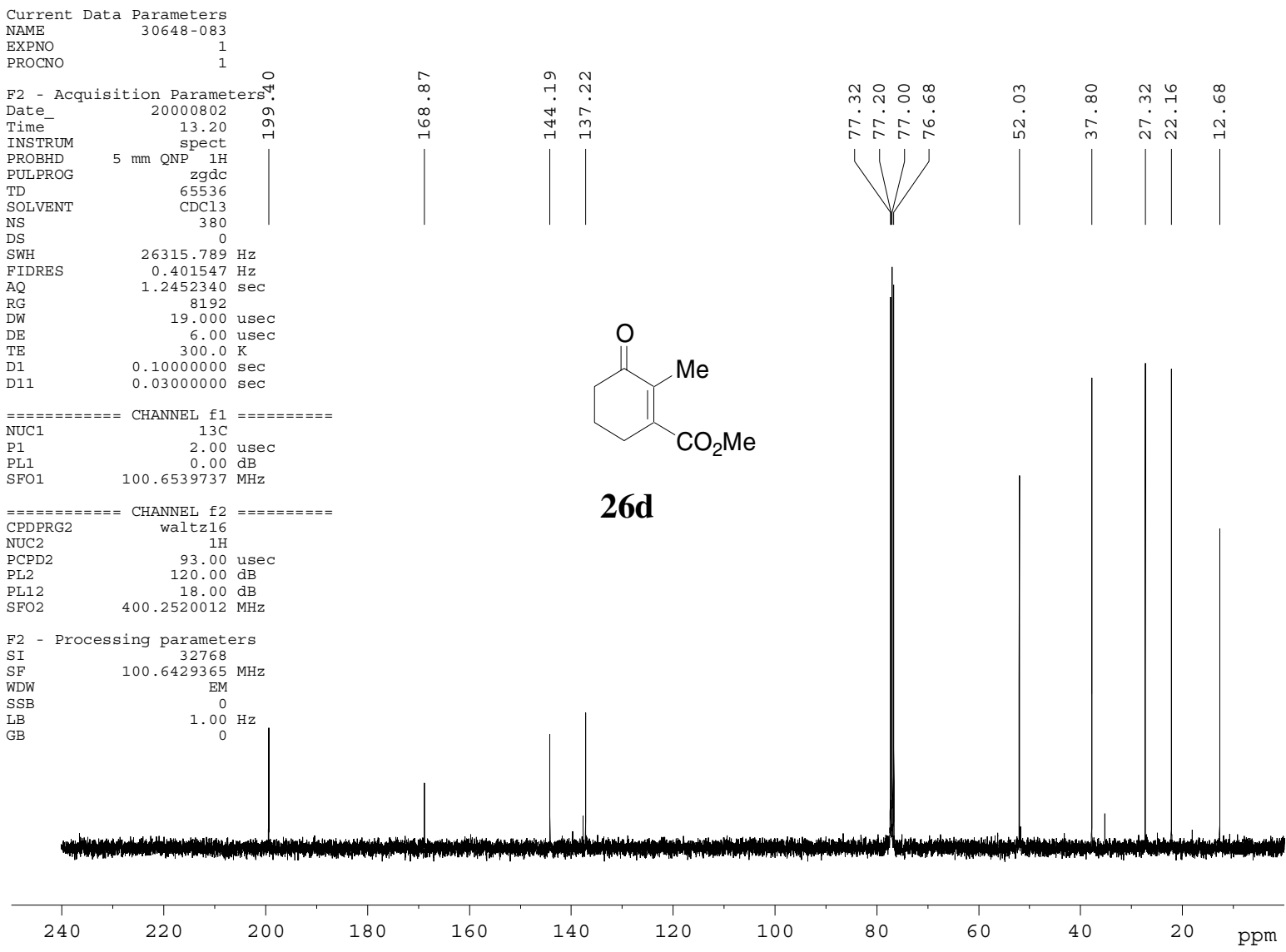

